\title{
EFEITO DA LIBERAÇÃO AUMENTATIVA NO CAMPO DE Pseudophilothrips ichini NO DESENVOLVIMENTO DE Schinus terebinthifolius
}

\author{
Luizimir Eduardo Furmann**, José Henrique Pedrosa-Macedo**, Jim P. Cuda***, \\ Marcelo Diniz Vitorino**** \\ * Acadêmico de Eng. Florestal, UFPR - luizimir@hotmail.com \\ ** Eng. Florestal, Dr., Setor de Ciências Agrárias, UFPR - johpema@netpar.com.br \\ *** Entomologista, Entomology and Nematology Department, University of Florida - jcuda@mail.gnv.iflas.ufl.edu \\ **** Eng. Florestal, Dr., Depto. de Engenharia Florestal, FURB - diniz@furb.br
}

Recebido para publicação: 20/04/2004 - Aceito para publicação: 20/06/2005

\begin{abstract}
Resumo
Efeito da liberação aumentativa no campo de Pseudophilothrips ichini no desenvolvimento de Schinus terebinthifolius. Experimentos de campo foram conduzidos com Pseudophilothrips ichini (Hood), 1949 (Thysanoptera: Tubulifera: Phlaeothripidae) por dois anos, para determinar o dano na produção de brotação na aroeira, Schinus terebinthifolius Raddi (Anacardiaceae). O primeiro experimento com 40 mudas de aroeira eqüiâneas foi executado entre agosto de 2001 e maio de 2002. O tratamento-testemunha $(\mathrm{n}=20)$ foi pulverizado com solução aquosa $(1 \%$ i.a. $)$ do inseticida Deltametrina $25 \mathrm{CE}$; o outro recebeu infestações forçadas de 400 espécimes, adultos e ninfas. $\mathrm{O}$ segundo experimento, de julho de 2002 a maio de 2003, foi realizado com 60 mudas, submetidas a três tratamentos. Vinte plantas foram infestadas artificialmente com 800 espécimes, adultos e ninfas $(\mathrm{n}=40$ por planta). No segundo grupo de mudas $(\mathrm{n}=20)$, ocorreu infestação natural. $\mathrm{O}$ terceiro grupo $(\mathrm{n}=20)$ foi pulverizado a cada 15 dias para excluir $P$. ichini. A perda de biomassa nos experimentos foi de $20 \%$ no primeiro e $62 \%$ no segundo; a infestação natural causou perda de $22 \%$. Os resultados mostraram que $P$. ichini é um potencial agente de controle da aroeira.

Palavras-chave: Aroeira, eficácia de campo, redução de biomassa, thrips.
\end{abstract}

\begin{abstract}
Pseudophilothrips ichini rising release in field and its effects on development of Schinus terebinthifolius. A manipulative field experiment replicated over a 2-year period was conducted with Pseudophilothrips ichini (Hood), 1949 (Thysanoptera: Tubulifera: Phlaeothripidae) in order to determinate damage on shoot production of its host Brazilian peppertree, Schinus terebinthifolius Raddi (Anacardiaceae). In the first trial, conducted from August 2001 to May 2002, 40 Brazilian peppertree seedlings of the same age, class and size were planted and randomly assigned by two treatment groups. Half of the plants $(n=20)$ were sprayed with $1 \%$ ai of Deltamethrin $25 \mathrm{CE}$, the other half was manually infested with 400 thrips, adults and larvae ( $\mathrm{n}=20$ per plant). 60 Brazilian peppertree seedlings subjected to three treatments constituted the second experiment, conducted from July 2002 to May 2003 at the same location. Twenty plants were artificially infested with 800 thrips adults and larvae ( $\mathrm{n}=40$ per plant). A second group of 20 seedlings suffered natural infestation. The third group of 20 seedlings was sprayed with insecticide each 15 days to exclude $P$. ichini. In the first experiment biomass of Brazilian peppertree was reduced by $20 \%$ in plants artificially infested with $P$. ichini whereas a $62 \%$ reduction rate was observed in the second experiment. Plants naturally infested with the thrips in the second experiment exhibited a $22 \%$ loss in biomass in relation to the control group. The experiment demonstrated $P$. ichini as a potential biological control agent of Brazilian peppertree.

Keywords: Brazilian peppertree, field efficacy, biomass reduction, thrips.
\end{abstract}




\section{INTRODUÇÃO}

Desde o início do século XIX, plantas nativas do Brasil têm sido introduzidas em outros países. A aroeira, Schinus terebinthifolius Raddi (Anacardiaceae) foi, deliberadamente, introduzida no Estado da Flórida como planta ornamental e continua se propagando, embora poucas plantas sejam utilizadas com essa proposta atualmente. Nesse Estado, a aroeira tem invadido e formado denso aglomerado em pastagens de baixo manejo, em terras agrícolas abandonadas ao lado de rodovias, às margens de canais e plantios de Pinus (Woodal, 1982; Langeland \& Burks, 1998). A sua rápida dispersão é ameaça de destruição do ecossistema natural do Parque Nacional de Everglades, além de cobrir cerca de 284 mil hectares (700.000 acres) no sul da Flórida (Ferriter, 1997; Langeland \& Burks, 1998). Na origem, isso é, na América do Sul (Argentina, Brasil, Paraguai e Uruguai), a aroeira é uma planta em equilíbrio devido à presença de seus inimigos naturais.

Nas Bahamas, a aroeira tem invadido grandes áreas da Grand Bahamas, New Providence e muitas outras ilhas. Essa planta também escapou ao cultivo nas Bermudas, onde os custos financeiros de controle periódico para a proteção da vegetação nativa estão se tornado elevados. No Havaí, a aroeira foi declarada, em 1978, como planta nociva, tendo se estabelecido em mais de 50.000 hectares nas partes mais secas das quatro principais ilhas do arquipélago. A rápida disseminação da aroeira em Queensland, na Austrália, criou cogitações para um programa de controle biológico também nesse país (Bennet et al., 1991).

De acordo com Morton (1979), a aroeira não é uma ameaça apenas à flora nativa de locais onde foi introduzida, mas também ao homem e animais domésticos ou não, podendo ocasionar problemas de saúde. Muitas são as pessoas sensíveis ao contato com a resina ou seiva da aroeira, contato este que pode ocasionar lesões, erupções severas e desconforto intenso. O fruto verde, se ingerido, pode ser fatal para cavalos, e o maduro, se comido em grande quantidade, causa intoxicação em algumas espécies de pássaros (Ewel, 1978).

Durante o florescimento da aroeira na Flórida, que ocorre por quase todo o ano, acredita-se que um cheiro proveniente das inflorescências causa congestão nasal, irritação dos olhos, dor de cabeça, espirros e dificuldade de respiração nas pessoas (Morton, 1978).

Devido ao seu pequeno porte, a aroeira é indicada para a arborização de ruas estreitas e sob fios elétricos, apesar de poder causar alergia a pessoas sensíveis que entram em contato com as folhas. As flores são melíferas. É uma das espécies mais procuradas pela avifauna, sendo, portanto, útil nos reflorestamentos heterogêneos destinados à recomposição de áreas degradadas, de preservação permanente. Trata-se de uma espécie perenifólia, heliófita e pioneira, comum em beira de rios, córregos e em várzeas úmidas de formações secundárias; contudo, cresce também em terrenos secos e pobres. É amplamente disseminada por pássaros, o que explica a sua boa regeneração natural. Sua dispersão é ampla, ocorrendo desde a restinga até a Floresta Ombrófila Mista (Lorenzi, 2000).

O entomologista explorador N. L. Krauss iniciou, na década de 1950, o levantamento de inimigos naturais e os estudos sobre controle biológico da aroeira, cujas pesquisas prosseguiram no Estado Norte Americano do Havaí. De um total de 30 espécies de insetos associados à aroeira e plantas botanicamente próximas, apenas três foram selecionadas para introdução da quarentena no Havai entre 1954 e 1961: Lithraeus (=Bruchus) atronotatus Pic (Coleoptera: Bruchidae), Epismus utilis Zimmerman (Lepidoptera: Tortricidae) e Crasimorpha infuscata Hodges (Lepidoptera: Galechiidae). Apenas os dois primeiros conseguiram se estabelecer, causando danos secundários que não diminuíram o crescimento, nem a agressividade da planta (Bennett et al., 1989). Uma quarta espécie Pseudophilothrips ichini Hood (Thysanoptera: Phlaeothripidae) (Liothrips ichini) foi investigada subseqüentemente por Garcia (1976), que relatou a intensidade e o tipo de dano que esse inseto causa na aroeira, indicando também uma possível especificidade a essa planta.

A busca de inimigos naturais da aroeira no Brasil iniciou-se na década de 90 . Uma centena de artrópodes associados foram encontrados, no entanto apenas cinco espécies - Calophya terebinthifolii Burkhard, 2000 (Homoptera: Psyllidae), Episimus utilis Zimmerman (Lepidoptera: Tortricidae), Heteroperreyia hubrichi Malaise, 1955 (Hymenoptera: Pergidae), Pseudophilothrips ichini, Apocnemidophorus pipitzi (Faust, 1886) (Coleoptera: Curculionidae) - demonstram potencial como futuros agentes de controle biológico da aroeira. O objetivo deste trabalho foi registrar o dano causado 
pela liberação aumentativa da espécie $P$. ichini e dimensionar a conseqüente perda de biomassa da aroeira.

\section{MATERIAL E MÉTODOS}

Os experimentos foram realizados na Fazenda Guajuvira localizada em Araucária (PR), Região Metropolitana de Curitiba, Primeiro Planalto Paranaense, cujas coordenadas geográficas são: 49 $29^{\prime} 37^{\prime}$, W, $25^{\circ} 09^{\prime} 22^{\prime}$ 'S e altitude de $940 \mathrm{~m}$. De acordo com Carpanezzi et al. (1986), o Primeiro Planalto do Paraná é caracterizado por altitudes entre 650 a $1100 \mathrm{~m}$, e pela Floresta Ombrófila Mista e campos submontanos. O sítio é exposto e inclinado para o sul, o solo é latossolo argiloso e tinha sido usado para fins agrícolas.

Os experimentos tiveram como objetivos a verificação e o dimensionamento do impacto causado na perda de biomassa. Chamou-se de Primeiro Experimento de campo aquele conduzido com dois tratamentos "A1" $(\mathrm{n}=20)$ e "B1" $(\mathrm{n}=20)$, ou seja, 40 mudas de aroeira - Schinus terebinthifolius var. raddianus (Anacardiaceae). Ele foi conduzido de agosto de 2001 a maio de 2002. O tratamento testemunha "B1" $(\mathrm{n}=20)$ foi pulverizado, quando necessário, com o inseticida Deltametrina $25 \mathrm{CE}(1 \%$ i.a.), e o tratamento "A1" recebeu duas infestações forçadas $(n=200)$ de adultos e ninfas, totalizando 400 espécimes. Com base nos resultados desse primeiro experimento, delineou-se o segundo, que foi realizado entre julho de 2002 e maio de 2003, no mesmo local e condições similares. Nele usaram-se três tratamentos: "A2", "B2" e "C2", cada um com 20 mudas. O tratamento infestado "A2" recebeu no total 800 espécimes, adultos e ninfas, isto é, recebeu duas infestações forçadas de 400 espécimes (adultos + ninfas). O segundo tratamento, "B2" $(\mathrm{n}=20)$, deixou-se para ocorrência de infestação natural. O tratamento-testemunha "C2" $(\mathrm{n}=20)$ foi pulverizado vinte vezes com o inseticida Deltametrina $25 \mathrm{CE}$ (1\% i.a.), isto é, a cada 15 dias, para evitar infestação. Em ambos os experimentos, o tamanho de cada parcela (tratamento) foi de $64,32 \mathrm{~m}^{2}$.

As 40 mudas do Primeiro Experimento, parcelas "A1" e "B1", foram preparadas com sementes de $S$. terebinthifolius var. raddianus procedentes da localidade de Borda do Campo, município de Quatro Barras, Paraná. Quando foram plantadas, tinham a idade de 460 dias e $50 \mathrm{~cm}$ de altura. Para preparar as mudas para o segundo experimento, foram coletadas sementes de uma única matriz existente na cidade de Balsa Nova, Paraná. A semeadura ocorreu em 5 de abril de 2001, e essas mudas foram repicadas em sacos plásticos $(6 \times 20 \mathrm{~cm})$ em 5 de outubro de 2001, no Arboreto Juvevê (SCA/UFPR). Com a idade de 448 dias e altura de $20 \mathrm{~cm}$, as 60 mudas foram plantadas em três parcelas, "A2", "B2" e "C2", na Fazenda Guajuvira, município de Araucária, Paraná, no dia 27 de julho de 2002.

Para obter o número de adultos e ninfas necessário, foram feitas várias coletas de Pseudophilothrips ichini no campo em Almirante Tamandaré (Juruqui), em Curitiba (Jardim Zoológico Municipal), em Campo Largo (Museu do Mate e perímetro urbano) e em Balsa Nova (perímetro urbano). Todos esses municípios estão localizados na Região Metropolitana de Curitiba.

Para dimensionar a perda de biomassa, cortaram-se as mudas do primeiro e segundo experimentos, respectivamente, em maio de 2002 e maio de 2003. Os pesos encontrados nas testemunhas "B1" e "C2" foram estabelecidos como padrão de crescimento da biomassa. As diferenças foram chamadas de "perda de biomassa" tanto no primeiro como no segundo experimento, mesmo no tratamento de infestação natural.

\section{RESULTADOS E DISCUSSÃO}

O primeiro experimento durou 242 dias. O tratamento "B1" produziu $61,5 \pm 15,4 \mathrm{~g} / \mathrm{planta}(\mathrm{n}=$ 20), totalizando $1.230,7 \mathrm{~g}(100 \%)$ de biomassa, somente dimensionadas as partes aéreas das plantas, enquanto que o tratamento "A1" produziu 43,3 $\pm 10,7 \mathrm{~g} /$ planta $(\mathrm{n}=20)$, totalizando $865,3 \mathrm{~g}(70 \%)$, ou seja, 365,4 g (30\%), 1,4 vezes a menos do que a testemunha. Esse valor representa o dano causado pela infestação forçada de thrips somada a uma infestação natural desconhecida de thrips e de outros artrópodes associados à aroeira (Figura 1). Os estudos comparativos demonstraram que o dano causado por thrips ( $P$. ichini) em mudas de aroeira (S. terebinthifolius var. Raddianus) atinge uma perda de biomassa variando entre 22 e $62 \%$. 
Após 302 dias, em maio de 2003, foram cortadas as mudas dos três tratamentos (“A2", "B2” e "C2") do segundo experimento que tinha sido instalado em agosto de 2002. A mensuração da biomassa resultou no seguinte: o tratamento " $\mathrm{C} 2$ " teve uma produção média de $21,6 \pm 7,6 \mathrm{~g} / \mathrm{planta}(\mathrm{n}=20)$, ou seja, uma produção total da parcela de 432,8 g (100\%) de biomassa, enquanto que o tratamento da parcela “A2" teve uma produção média de 8,2 \pm 3,9 g/planta de biomassa, o que vem a corresponder a uma produção de 2,6 vezes menor do que a da testemunha. A sua produção total de biomassa foi de $163,6 \mathrm{~g}$ $(37,8 \%)$, registrando uma perda de 269,2 g $(62,2 \%)$ em relação à testemunha "C2" (Figura 1). O tratamento "B2" teve uma produção média de $16,8 \pm 5,8 \mathrm{~g} /$ planta $(\mathrm{n}=20)$, correspondendo a 1,3 vezes menor do que a da testemunha. A sua produção total de biomassa foi de 336,7 g (77,8\%), ou seja, teve uma perda de 96,1 g (22,2\%) em relação à testemunha, tratamento "C2" (Figura.1).

Desse modo, o tratamento "B2" comprovou e justificou a necessidade de sua realização, pois demonstrou claramente que a ocorrência de infestação natural de thrips e outros artrópodes causou a perda de 1/3 da biomassa, conforme a avaliação estatística acima. Por outro lado, observa-se que uma infestação forçada, nas condições desse experimento, causou a perda de biomassa em $2 / 5(40 \%) \mathrm{em}$ relação à testemunha.

Pode-se verificar que a diferença no número de espécimes colocados para a infestação entre os dois experimentos é relevante, pois ocorreu uma diminuição maior na biomassa no segundo experimento em relação ao primeiro, decorrente da introdução do dobro de espécimes/planta. Por outro lado, observase que entre um ano e outro ano há diferentes níveis de infestação natural. Basta observar que, no primeiro experimento realizado, a perda de biomassa foi de $30 \%(365,4 \mathrm{~g})$ num período menor (242 dias), enquanto que no segundo experimento a perda de biomassa foi de $22 \%(96,10 \mathrm{~g})$ num período maior (302 dias).

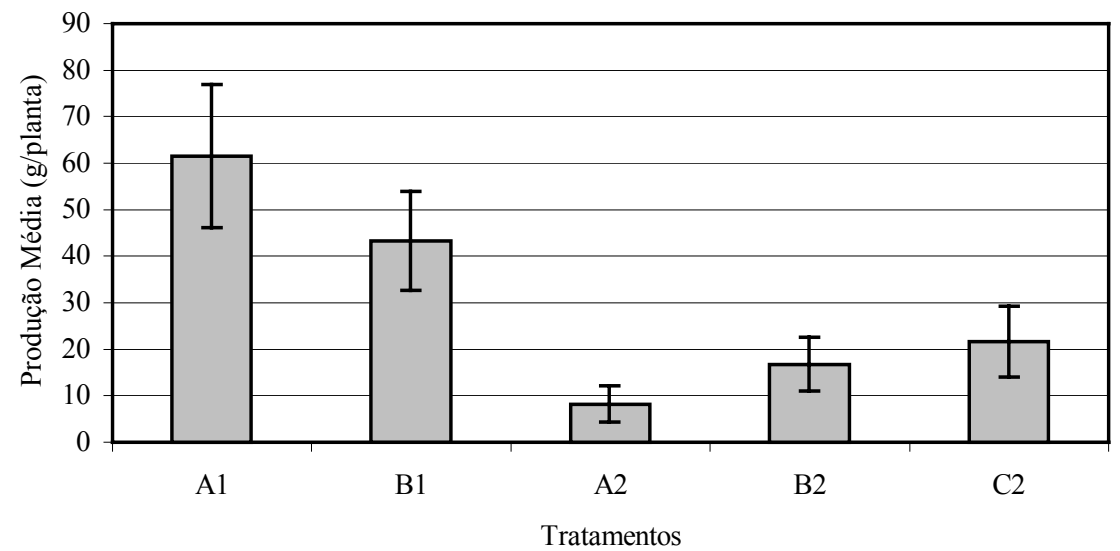

Figura. 1. Perda de biomassa causada por ataque de Thrips - Pseudophilothrips ichini Hood, 1949 (Thysanoptera: Phloeothripidae) em mudas de aroeira - Schinus terebinthifolius Raddi (Anacardiaceae) durante 242 dias no Experimento I (A1 e B1) e durante 302 dias no Experimento II (A2, B2 e C2) - Fazenda Guajuvira, Araucária, Paraná, 2001.

Figure 1. Biomass loss caused by the Thrips - Pseudophilothrips ichini Hood, 1949 (Thysanoptera: Phloeothripidae) on Brazilian Peppertree seedlings - Schinus terebinthifolius Raddi (Anacardiaceae) by 242 days in Experiment I (A1 and B1) and by 302 days in Experiment II (A2, B2 and C2) - Guajuvira Farm, Araucaria, Paraná, 2001.

\section{CONCLUSÕES}

Conclui-se que a infestação forçada aumentou a perda de biomassa em dois quintos, ou seja, em $40 \%$, abrangendo um maior número de brotações. Finalmente, conclui-se que a supressão da brotação afetará a produção futura de frutos. 


\section{AGRADECIMENTOS}

Ao South Florida Water Management District e ao Florida Department of Environmental Protection, pelo suporte financeiro. À FUPEF do Paraná, pela gestão do recurso financeiro. Ao IEL/FUPEF, pela concessão de bolsa. Ao colega Prof. Dr. Julio Medal e à Sra. Judy Gilmore, do Departamento de Entomologia e Nematologia da Universidade da Flórida, pela cooperação. À Prof ${ }^{a}$. Dra. Márcia Cristina Mendes Marques e à Prof ${ }^{\mathrm{a}}$. Marion do Rocio Foerster Avanci, pela revisão do texto, e à Engenheira Florestal Lorena Stolle, pela elaboração do gráfico. Aos colegas bolsistas Edgard Alfredo Bredow, Thiago Luiz Finger Granemann e Luiz Alessandro Scapini, pela colaboração.

\section{REFERÊNCIAS}

BARKLEY, F. A. A Study of Schinus L. Lilloa Revista de Botanica, Tucuman, t.28, 110p., 1957.

BENNET, F. D.; HABECK, D. H. Brazilian Peppertree Prospects for Biological Control in Florida. In: SYMPOSIUM ON EXOTIC PEST PLANTS, Proceedings of the..., 1991

CARPANEZZI, A. A.; Zoneamento ecológico para plantios florestais no Estado do Paraná. Colombo: EMBRAPA-CNPF, 1986. 89p. (Documentos, 17).

EWEL, J. Ecology of Schinus. Schinus - Technical Proceedings of Techniques for Control of Schinus in South - Florida: A Workshop for Natural Area Manager's. Sanibel, Florida,1978.

FERRITER, A. Brazilian Pepper Management Plan for Florida. Florida Exotic Pest Plant Council's., 1997.

GARCIA, C. A. Biologia e Aspectos da Ecologia e do Comportamento Defensivo de Liothrips ichini Hood, 1949 (Thysanoptera, Tubulifera). Curitiba-PR, 1976. Dissertação (Mestrado em Entomologia) Setor de Ciências Biológicas, Universidade Federal do Paraná.

JOLY, A. B. Botânica: Introdução à taxonomia vegetal. 7.ed., Nacional. 1985.

LANGELAND, K. A: BURKS, K. C. Identification \& Biology of Non-Native Plants in Florida's Natural Areas. Gainesville-FL: University of Florida, 1998.

LORENZI, H. Árvores Brasileiras. manual de identificação de espécies arbóreas. 3. ed.. Nova Odessa: Plantarum, 2000.

MORTON, J. F. Brazilian Peppertree - Its Impact on People, Animals, and the environment. Econ. Botany, 1979.

WOODALL, S. Physiology of Schinus. In: Schinus Technical Proceedings of Techniques for Control of Schinus in South- florida: A Workshop for Natural Area manager's. Sanibel- FL: the Sanibel- Captiva Conservation Foundation, Inc., 1978.

WORKMAN, R. W. History of Schinus in Florida. In: Schinus Technical Proceedings os Techniques for Control of Schinus in South-Florida: A Workshop for Natural Area Manager's. Sandibel-FL: The Sandibel-Captiva Conservation Foundation, Inc., 1978. 